\title{
A randomized controlled trial of the GLP-1 receptor agonist dulaglutide in primary polydipsia
}

\author{
Bettina Winzeler, ${ }^{1,2}$ Clara O. Sailer, ${ }^{1,2}$ David Coynel, ${ }^{3,4}$ Davide Zanchi,, ${ }^{5,6}$ Deborah R. Vogt, ${ }^{1,2,7}$ Sandrine A. Urwyler, ${ }^{1,2}$ \\ Julie Refardt, ${ }^{1,2}$ and Mirjam Christ-Crain ${ }^{1,2}$ \\ 'Department of Endocrinology, Diabetology and Metabolism, University Hospital Basel, Basel, Switzerland. ${ }^{2}$ Department of Clinical Research, University of Basel, Basel, Switzerland. ${ }^{3}$ Division of Cognitive \\ Neuroscience, Department of Psychology and ${ }^{4}$ Transfaculty Research Platform, University of Basel, Basel, Switzerland. ${ }^{5}$. Hoffmann-La Roche, Roche Innovation Centre Basel, Basel, Switzerland. ${ }^{6}$ Stanford \\ University Graduate School of Business, Stanford, California, USA. ${ }^{7}$ Clinical Trial Unit, University of Basel and University Hospital Basel, Basel, Switzerland.
}

BACKGROUND. Primary polydipsia, characterized by excessive fluid intake, carries the risk of water intoxication and hyponatremia, but treatment options are scarce. Glucagon-like peptide 1 (GLP-1) reduces appetite and food intake. In experimental models, GLP-1 has also been shown to play a role in thirst and drinking behavior. The aim of this trial was to investigate whether GLP-1 receptor agonists reduce fluid intake in patients with primary polydipsia.

\begin{abstract}
METHODS. In this randomized, double-blind, placebo-controlled, 3-week crossover trial, 34 patients with primary polydipsia received weekly dulaglutide ( $1.5 \mathrm{mg}$, Trulicity) in one treatment segment and placebo ( $0.9 \%$ sodium chloride) in the other. During the last treatment week, patients attended an 8-hour evaluation visit with free access to water. The primary endpoint was total fluid intake during the evaluation visits. Treatment effects were estimated using linear mixed-effects models. In a subset of 15 patients and an additional 15 matched controls, thirst perception and neuronal activity in response to beverage pictures were assessed by functional MRI.
\end{abstract}

RESULTS. Patients on dulaglutide reduced their fluid intake by $490 \mathrm{~mL}(95 \% \mathrm{Cl}:-780,-199 ; P=0.002)$, from $2950 \mathrm{~mL}$ (95\% Cl: 2435,3465$)$ on placebo to $2460 \mathrm{~mL}$ (95\% Cl: 1946, 2475) on dulaglutide (model estimates), corresponding to a relative reduction of $17 \%$. Twenty-four-hour urinary output was reduced by $-943 \mathrm{~mL}$ ( $95 \% \mathrm{Cl}:-1473,-413 ; P=0.001$ ). Thirst perception in response to beverage pictures was higher for patients with primary polydipsia than for controls, and lower for patients on dulaglutide versus placebo, but functional activity was similar among groups and treatments.

CONCLUSIONS. GLP-1 receptor agonists reduce fluid intake and thirst perception in patients with primary polydipsia and could therefore be a treatment option for these patients.

TRIAL REGISTRATION. Clinicaltrials.gov NCT02770885.

FUNDING. Swiss National Science Foundation (grant 32473B_162608); University Hospital and University of Basel; Young Talents in Clinical Research grant from the Swiss Academy of Medical Sciences and the Gottfried \& Julia Bangerter-Rhyner Foundation; Top-up Grant from the PhD Programme in Health Sciences, University of Basel.

\section{Introduction}

Primary polydipsia is characterized by exaggerated thirst perception and excessive drinking $(1,2)$. This syndrome has most often been described in patients with psychiatric disorders (3) but seems increasingly prevalent in health-conscious people who voluntarily change their drinking habits with the aim of improving their well-being (4). However, high fluid intake is not always healthy

Authorship note: BW and COS are co-first authors.

Conflict of interest: The authors have declared that no conflict of interest exists. Role of funding source: The funders of the study had no role in the study design, data collection, data analysis, data interpretation, or writing of the report. The first and last authors had access to all the data and had final responsibility for the decision to submit for publication.

Copyright: @ 2021, American Society for Clinical Investigation.

Submitted: June 1, 2021; Accepted: August 31, 2021; Published: September 2, 2021.

Reference information: J Clin Invest. 2021;131(20):e151800.

https://doi.org/10.1172/JCl151800. or harmless. In fact, it may lead to life-threatening water intoxication, hyponatremia, and cerebral edema (1). In light of these consequences, primary polydipsia merits careful evaluation and appropriate treatment. Unfortunately, few treatment options exist (5). Behavioral therapy is often ineffective, given the compulsive component of this disorder and the persistent thirst perception $(6,7)$. In patients with psychiatric disorders, medications such as clozapine, risperidone, or lithium have been proposed in case reports or case series and may modulate drinking behavior by controlling psychotic symptoms $(8,9)$. However, the unfavorable side effects, such as weight gain and diabetes mellitus, render these medications unsuitable outside of the acute psychiatric setting (10). So far, there are no published randomized, controlled trials investigating pharmacological treatment options for fluid intake in primary polydipsia.

The hormone glucagon-like peptide 1 (GLP-1) is released in response to food intake $(11,12)$ and is involved in the central reg- 


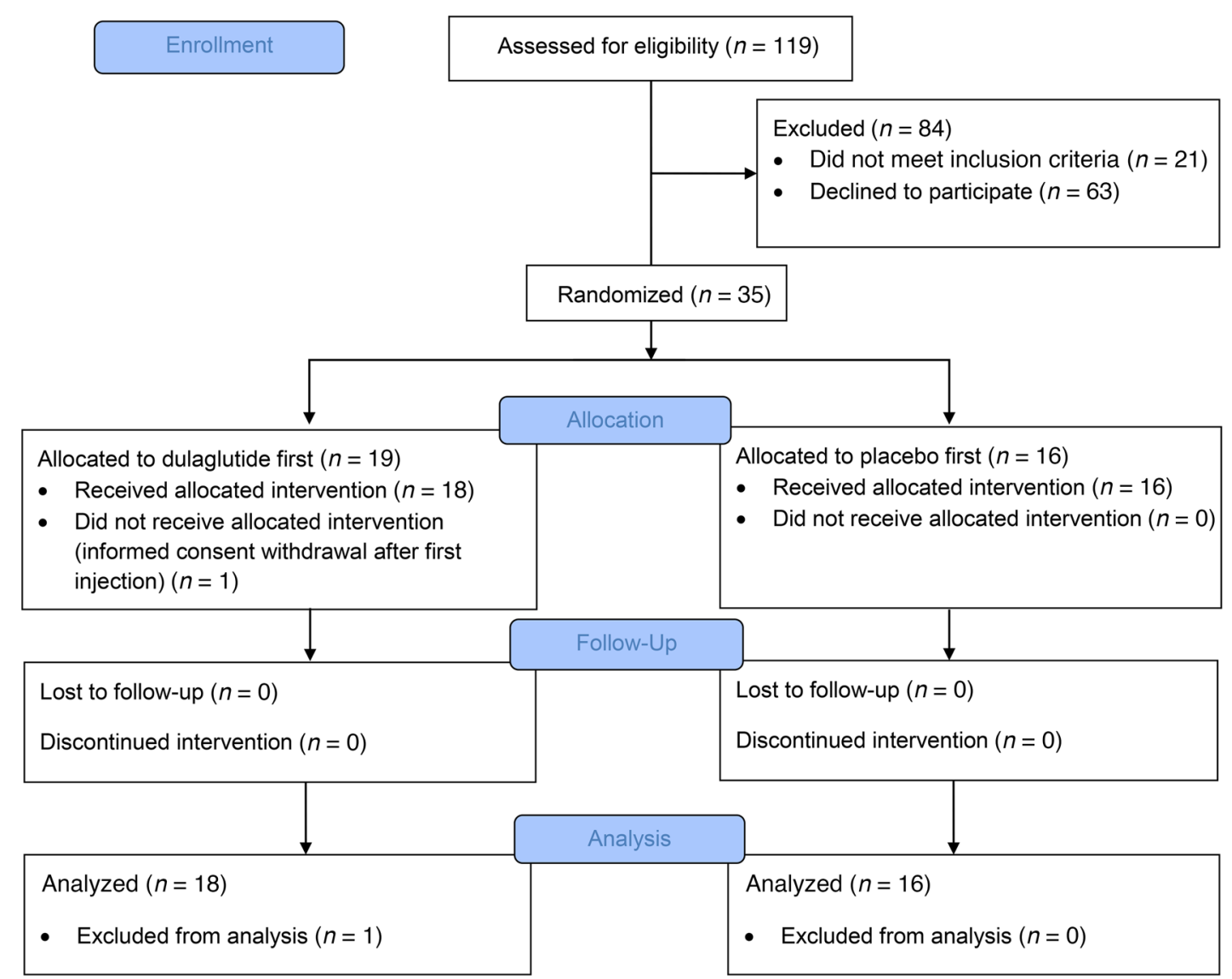

Figure 1. Consort flow diagram. Reconstructed CONSORT diagram for the randomized, controlled trial.

ulation of appetite and energy intake $(13,14)$. GLP-1 receptor agonists (GLP-1 RAs) are widely used to treat diabetes mellitus and obesity (15). Emerging evidence suggests that the satiating properties of GLP-1 are not limited to food intake, but may also have an impact on thirst and drinking behavior (16-19). In fact, neurons of the lamina terminalis - a key brain structure for sensing thirst and regulating water balance $(20,21)$ - express GLP-1 receptors and are believed to confer satiety of thirst during fluid ingestion (22). In rats, McKay et al. observed a reduction of fluid intake following GLP-1 RA treatment, which was independent of food intake (23). In humans, we have recently shown that the GLP-1 RA dulaglutide tends to reduce fluid intake and lowers 24-hour urinary output in healthy volunteers (24).

GLP-1 may regulate not only homeostatic thirst, but might also have a role in hedonic control and the pathophysiology of addiction $(25,26)$. The compulsive or addictive components of primary polydipsia may therefore be targeted by GLP-1 RAs.

The aim of this trial was to investigate whether a GLP-1 RA as compared with placebo reduces fluid intake in patients with primary polydipsia. Second, we aimed to explore the effect of dulaglutide versus placebo on thirst perception, brain activity, and resting-state functional connectivity.

\section{Results}

Baseline characteristics. Between 2016 and 2019, a total of 35 patients were enrolled in the trial and randomly assigned to groups (Figure 1). One patient withdrew informed consent after the first study drug injection because of adverse events, i.e., recurrent vomiting (unblinding showed that the patient had received dulaglutide). The other 34 patients, who all received the intended treatment and were analyzed, were predominantly female (67.6\%) and had a median (IQR) age of 29.5 years (26.0, 38.8). Fourteen (41.2\%) patients had psychiatric comorbidities and were labeled as having "psychogenic polydipsia," whereas the remaining 20 patients were labeled as having "habitual polydipsia." At baseline, the median (IQR) reported fluid intake was $4500 \mathrm{~mL}$ per day $(3600,5000)$. Baseline characteristics are shown in Table 1. The baseline characteristics before placebo treatment according to treatment sequence, i.e., placebo or dulaglutide first, were similar between the 2 groups. The only apparent difference was an increase in diastolic blood pressure before the beginning of the placebo treatment in patients who received dulaglutide first. However, this patient group also had higher diastolic blood pressure compared with the other randomized patient group at the beginning of the first treatment (see Supplemental Table 1; supplemental material available online with this article; https://doi. org/10.1172/JCI151800DS1).

Fluid intake and 24-hour urinary output. The estimated total fluid intake during the evaluation visit in the placebo group was 2950 mL (95\% CI: 2435, 3465) versus 2460 mL (95\% CI: 1946, 2475) for patients on dulaglutide. Hence, patients on dulaglutide had an estimated reduction in fluid intake of $-490 \mathrm{~mL}$ (95\% CI: 


\section{Table 1. Baseline characteristics}

n

Age (yr), median (IQR)

34

Female sex, $n(\%)$

$29.5(26.0,38.8)$

$23(67.6)$

$11(32.4)$

Male sex, $n(\%)$

$34(100 \%)$

White race, $n(\%)$

$23.1(20.7,25.5)$

BMI $\left(\mathrm{kg} / \mathrm{m}^{2}\right)$, median (IQR)

$14(41.2)$

Psychogenic polydipsia, $n$ (\%)

$20(58.8)$

$14(41.2)$

Other comorbidities, $n(\%)$

$14(41.2)$

Smoking, $n(\%)$

Polydipsic characteristics

Reported daily fluid intake (mL), median (IQR)

$4500(3600,5000)$

24-hour urine output (mL), median (IQR)

Daytime voiding frequency, median (IQR)

$4700(3900,5600)$

$10(8,12)$

Drinking at night, $n(\%)$

$17(50)$

Nocturia, $n(\%)$

$-780,-199 ; P=0.002)$ compared with placebo (Figure 2A). This corresponds to a relative reduction of $17 \%$. Two-thirds of patients drank less on dulaglutide than when they were on placebo (Figure $2 \mathrm{~B}$ ). These findings were consistent in the per-protocol analysis (estimated mean difference [95\% CI] for dulaglutide versus placebo $-492 \mathrm{~mL}[-811,-173])$ and in the sensitivity analyses adjusting for adverse effects at the beginning of the evaluation visit $(-474 \mathrm{~mL}[-812,-136])$ or anytime during the evaluation visit $(-428 \mathrm{~mL}[-850,-6])$.

The estimated 24-hour urinary output for patients on dulaglutide was lower compared with placebo: $3591 \mathrm{~mL}$ (95\% CI: 2922, 4260 ) versus $4534 \mathrm{~mL}$ (95\% CI: 3865, 5203), with an estimated mean difference of $-943 \mathrm{~mL}$ (95\% CI: $1473,-413 ; P=0.001)$.

We found no evidence of a difference between patients with psychogenic versus habitual polydipsia with regard to total fluid intake or 24-hour urinary output (post hoc analyses, data not

shown). Body weight, median (IQR), remained stable during the study period (65.8 kg [55.3-77.5] and $65.3 \mathrm{~kg}$ [56.5-78.5] for dulaglutide and placebo, respectively).

Thirst and drinking behavior. Acute thirst perception at different time points of the evaluation visit was lower for patients on dulaglutide compared with placebo (Supplemental Figure 1). Reported thirst perception of the preceding weeks remained constant during treatment with dulaglutide and increased slightly with placebo treatment.

The reported daily fluid intake decreased for patients on dulaglutide from a median (IQR) of $4500[3625,5000] \mathrm{mL}$ to 3000 [2500, 3875] $\mathrm{mL}$, while it remained constant with placebo treatment, from 4000 [4000, 5000] $\mathrm{mL}$ to $4000[3500,5000] \mathrm{mL}$. The estimated, baseline-adjusted difference during the week preceding the evaluation visit between dulaglutide and placebo was -1257 mL (95\% CI: -1751, -764; $P<0.001$ ) (Figure 3A).

Self-reported daytime voiding frequency, median (IQR), decreased for patients on dulaglutide from $9.5(6.0,12.0)$ to 7.5 $(6.0,9.0)$, while we observed no obvious decrease with the placebo treatment, as the daytime voiding frequency only decreased from $10.0(6.5,12.0)$ to $9.5(7.0,11)$ (Figure $3 \mathrm{~B})$. The proportion of patients reporting drinking at night did not change, but nocturia decreased for patients on dulaglutide, while it slightly increased with placebo treatment (Figure 3C). Nocturia resolved in 7 of 19 patients on dulaglutide and in 1 of 16 patients on placebo.

Serum and urinary electrolytes. At the beginning of the evaluation visit, patients' serum sodium levels were in the normal range and did not differ between the dulaglutide and placebo groups (median [IQR]: $140 \mathrm{mmol} / \mathrm{L}$ [138; 141] versus $140 \mathrm{mmol} / \mathrm{L}$ [138; 141]). Twenty-four-hour urinary electrolyte levels were similar with dulaglutide and placebo treatments. Of note, our data provided no evidence of a difference in urinary sodium excretion between the treatment arms (estimated mean difference; 95\% CI: -8.67 mmol/L [-19.37, 1.82]; $P=0.12$ ) (Supplemental Table 2).

We evaluated the individual differences in sodium or urine osmolality changes during the evaluation visit while patients were
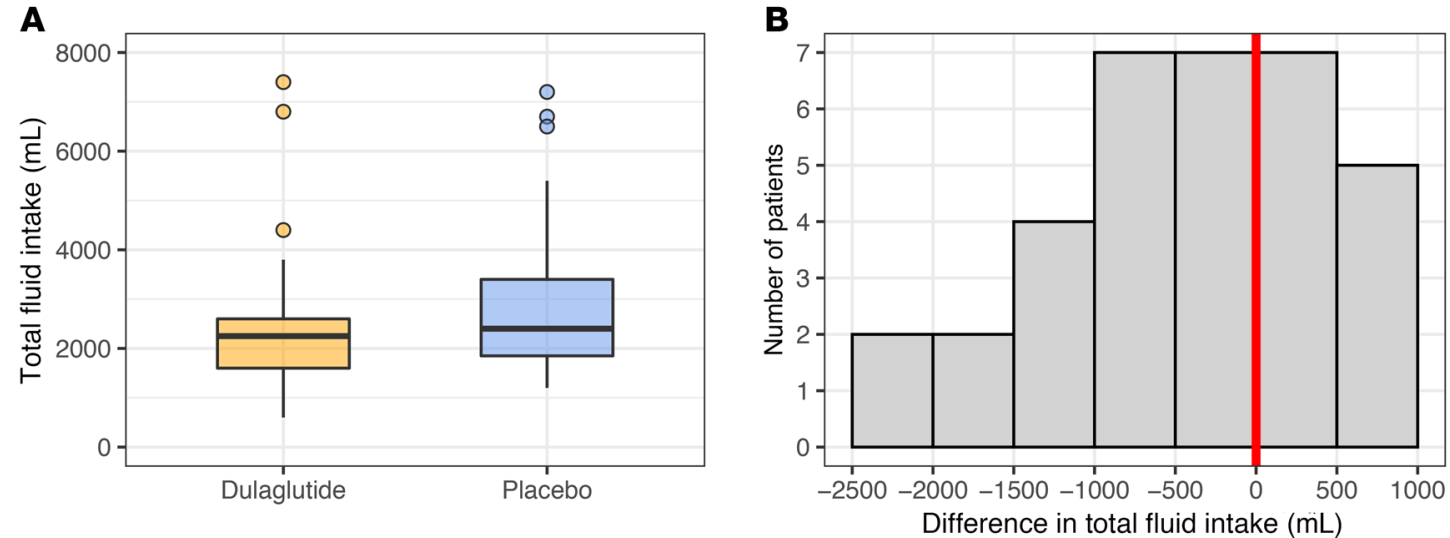

Figure 2. Observed total fluid intake during the evaluation visit for participants on dulaglutide or placebo. (A) Thick line indicates the median; box indicates the IQR; whiskers include all points within the range of 1.5 times the IQR; dots represent all points outside 1.5 times the IQR. Note that the figure shows descriptive summary statistics of the data ("raw data"), whereas we report the estimated means and the mean difference from statistical models in the results (linear mixed-effect model with trial arm as a single fixed effect and patient as a random effect). (B) Within-patients differences in total fluid/ water intake (within 8 hours) during the evaluation visit between treatment with dulaglutide versus placebo. Differences were calculated as the value in patients on dulaglutide minus the value when on placebo, hence, negative differences indicate a reduced fluid/water intake on dulaglutide, while positive values indicate an increased fluid/water intake on dulaglutide. 

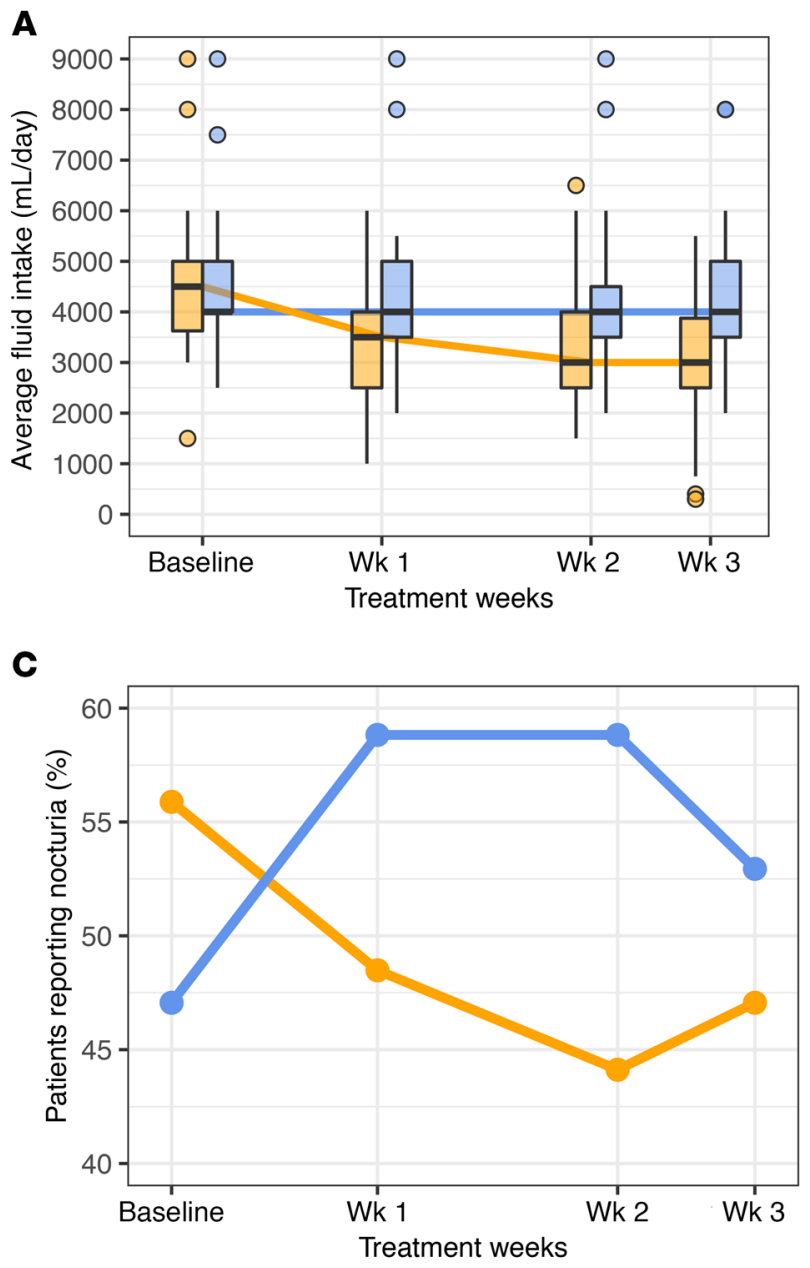

B

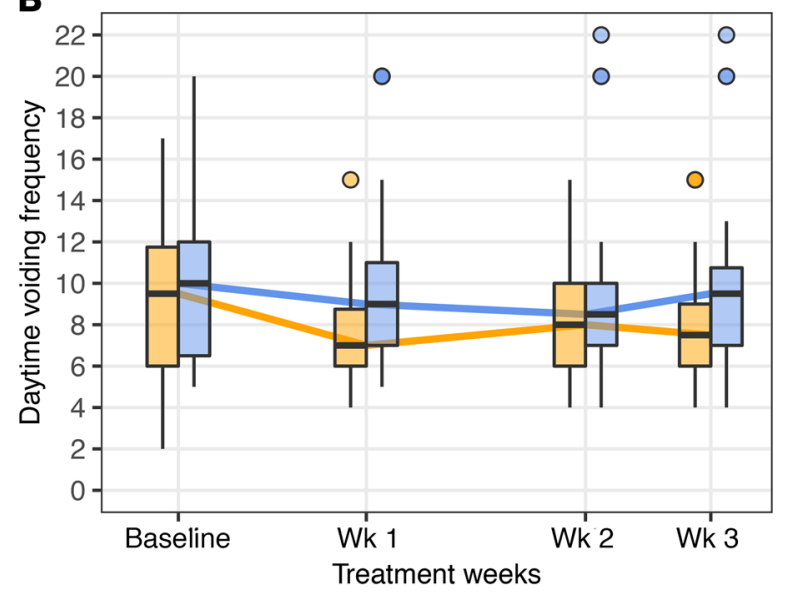

Treatment arm

Dulaglutide

Placebo

Figure 3. Time course of self-reported average daily fluid intake, daytime voiding frequency, and nocturia. (A) Self-reported average daily fluid intake during the preceding week for each study injection visit and both treatment arms. (B) Daytime voiding frequency per day during the preceding weeks for each study injection visit and both treatment arms. Thick line indicates the median; box indicates the IQR; whiskers include all points within the range of 1.5 times the IQR; dots represent all points outside 1.5 times the IQR. (C) Patients reporting nocturia during the preceding week for each study injection visit and both treatment arms. freely drinking and found a less pronounced decrease in these parameters for most patients on dulaglutide versus placebo (Supplemental Figures 2 and 3).

Quality of life. At baseline, i.e., before both treatment phases, patients indicated a moderate reduction in quality of life due to symptoms of polyuria and polydipsia (numeric rating scale [NRS]; median [IQR]:3.00 [0.25, 4.75] for dulaglutide and 3.0 [1.25, 5.00] for placebo). This decreased for both treatments, but was slightly stronger on dulaglutide compared with placebo (baseline-adjusted estimated mean difference, 95\% CI: $-0.9[-1.9,0.1] ; P=0.019)$.

The results from the SF-12 quality-of-life questionnaire did not show a clear change for patients on dulaglutide or placebo treatment (see Supplemental Results).

Adverse effects. Gastrointestinal side effects were more prevalent when patients were on dulaglutide compared with placebo and peaked during the first treatment week. On the morning of the evaluation visit, the number of patients reporting nausea was balanced between the treatment arms ( $n=2$ patients in each arm) (Supplemental Table 3 and Supplemental Figure 4).

Two patients developed mild hyponatremia during both evaluation visits. For the first patient (female, 56 years of age, on multiple medications including low-dose quetiapine), the minimum plasma sodium level on dulaglutide was $133 \mathrm{mmol} / \mathrm{L}$ versus 131 $\mathrm{mmol} / \mathrm{L}$ on placebo. In the second patient (healthy female, 24 years of age), the minimum plasma sodium level on dulaglutide was $132 \mathrm{mmol} / \mathrm{L}$ versus $133 \mathrm{mmol} / \mathrm{L}$ on placebo. Both patients had chronic, profound primary polydipsia (reported daily fluid intake of $5000 \mathrm{~mL}$ and $6000 \mathrm{~mL}$ and collected 24-hour urinary output of $6000 \mathrm{~mL}$ per day at baseline). The first patient showed a reduction of fluid intake (during the evaluation visit) from $6500 \mathrm{~mL}$ to $4400 \mathrm{~mL}$ on dulaglutide, whereas the second patient did not respond to dulaglutide (fluid intake of $6700 \mathrm{~mL}$ and $7400 \mathrm{~mL}$ on dulaglutide and placebo, respectively).

A few other adverse effects such as fatigue, headache, and mild upper respiratory tract infections were recorded during the study drug intervention. These adverse effects were similar between the treatment arms and were considered to be unrelated to the trial drug (data not shown).

Functional MRI results. The baseline characteristics of 15 patients with primary polydipsia from the mother study $(n=11$ females, median [IQR] age of 32 years [25, 39.5], $n=2$ left-handed) and an additional 15 matched controls enrolled in the functional MRI (fMRI) substudy are shown in Supplemental Table 4. Plasma sodium concentrations at the time of the fMRI sessions were in the mid-normal range and were similar for patients when on dulaglutide or placebo and for controls (data not shown).

The thirst ratings for patients were highest on placebo treatment when exposed to beverage pictures (NRS; median [IQR] 6, 6-6). Dulaglutide reduced thirst ratings both while patients viewed beverage pictures (NRS; median [IQR] 6, 4.5-6; $P=$ 


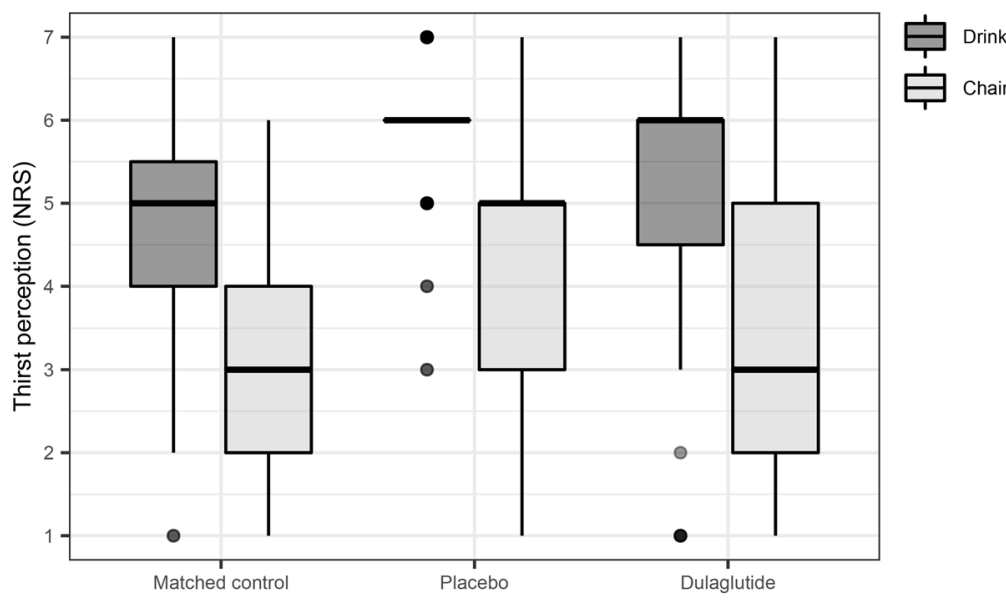

Figure 4. Thirst perception during the functional paradigm of the MRI visit. Selfreported thirst perception on a 7-point NRS for matched controls and patients on dulaglutide or placebo during the functional paradigm of the MRI visit. Thick line indicates the median; box indicates the IQR; whiskers include all points within the range of 1.5 times the IQR; dots represent all points outside 1.5 times the IQR. impact of dulaglutide in a real-life setting. In fact, the self-reported average daily fluid intake decreased by more than $30 \%$ (mean difference of $-1600 \mathrm{~mL}$ per day) on dulaglutide, which is in line with the assessed mean 24-hour urinary output reduction of $1000 \mathrm{~mL}$ per day. As a consequence, daytime voiding frequency and nocturia decreased on dulaglutide, and polydipsic patients felt less constrained by polyuria-polydipsia symptoms. Additionally, serum sodium and urinary osmolality showed a lesser decline throughout the day in most patients receiving dulaglutide, supporting the clinically relevant impact of reduced fluid intake with dulaglutide.

In a healthy individual, the regulation of thirst and drinking integrates both homeostatic and behavioral signals $(21,22,27)$, i.e., fluid intake is calibrated according to physiological need before osmolality and blood volume effectively change $(28,29)$. GLP- 1 seems to have a direct role in this regulatory circuit (30), as enriched GLP-1 receptor expression has been found in thirst-inhibitory neurons of the lamina terminalis 0.0017) and control pictures (NRS; median [IQR] 5 [3-5] versus 3 [2-5]; $P=0.0016)$. For matched controls, thirst ratings were higher while they were exposed to beverage pictures versus control pictures, but both ratings were lower as compared with patients with primary polydipsia (Figure 4).

The whole-brain analysis did not reveal any treatment-stimuli interaction effect in patients. The analysis of the main effect of treatment across both stimuli did not reveal any significant difference (Supplemental Figure 5, A and B). The analysis of the main effect of stimuli did not reveal any specific activation of regions linked to thirst regulation or motivation while participants were exposed to beverage pictures, not even in the subgroup of participants with a high state of thirst (Supplemental Figure 6, A and B).

When comparing controls and patients on placebo, we found no treatment: stimuli interaction effect (Supplemental Figure 7).

Resting-state functional connectivity in the reward network or the hypothalamus did not differ between dulaglutide and placebo treatments, nor between patients as compared with controls.

\section{Discussion}

Our results provide evidence that GLP-1 RAs have hypodipsic properties and lead to a reduction in fluid intake and thirst perception in patients with primary polydipsia, offering a pharmaceutical treatment option for these patients.

The hypodipsic effect of dulaglutide was independent of food intake and was not a consequence of altered body weight while on dulaglutide treatment, which is in line with data from studies in rodents and healthy volunteers $(23,24)$.

Dulaglutide decreased fluid intake on the evaluation visit by $17 \%$, which was more than the expected $13 \%$ reduction. Although we were not able to compare the efficacy of dulaglutide with other pharmacological treatments due to a lack of data (5), we consider the hypodipsic effect of dulaglutide to be clinically relevant. Given the short observation period of 8 hours and the artificial setting with a limited choice of beverages, the effect size of a $17 \%$ reduction may even underestimate the therapeutic
(22) - a key brain structure for water homeostasis (20). In rats, a polydipsic overdrinking phenotype was observed after ablation of these GLP-1 receptor-expressing thirst-inhibitory neurons (22). In our trial, dulaglutide had an important impact on thirst perception. Acute thirst ratings during the evaluation visit as well as during the thirst-craving task in the fMRI session were clearly lower in patients when on dulaglutide than on placebo, although we were not able to detect associated changes in functional brain activity. As an expression of an exaggerated thirst perception, patients (on placebo) versus matched controls scored higher thirst ratings irrespective of beverage or control pictures. Dulaglutide reduced patients' thirst ratings while exposed to control pictures within the range of ratings for matched controls. Importantly, polydipsic patients never reached thirst satiation, not even with free access to water. The sodium levels of participants were in the mid-to-normal range and did not differ between treatment arms or between patients and matched controls. Based on our results, the exaggerated thirst perception or desire to drink in patients with primary polydipsia seems to be uncoupled from osmolality and homeostatic factors.

Aside from the homeostatic aspects, drinking behavior is also influenced by psychological factors such as motivation and learning. The compulsive component of fluid intake in primary polydipsia shares features similar to those of other addictive behaviors such as excessive eating or illicit drug use $(25,31)$. Interestingly, a growing literature implies that GLP-1 is also involved in reward regulation and the pathophysiology of addiction (25), as GLP-1 receptors are expressed in brain areas related to reward processing (3234). Peripheral GLP-1 administration in humans has been shown to modulate functional brain activity in regions such as the amygdala, insula, caudate, putamen, and orbitofrontal cortex, supporting this hypothesis $(35,36)$. Therefore, we assume that the hypodipsic properties of dulaglutide in primary polydipsia may also be explained by modulation of addictive components of this condition. Our exploratory fMRI substudy did not enable us to reinforce this hypothesis. Specifically, we did not observe an altered resting state functional 
connectivity between homeostatic and reward-related brain areas with GLP-1 RA treatment, as previously demonstrated in healthy volunteers by Meyer-Gerspach et al. (37).

The most important complication of primary polydipsia is water intoxication and hyponatremia (38). In our trial, 2 patients experienced mild hyponatremia during the evaluation visits, with both patients receiving placebo and dulaglutide. In primary polydipsia, hyponatremia occurs when fluid intake exceeds urinary and insensible losses (38). Thus, the risk of hyponatremia increases in profound polydipsia and if the renal capacity to excrete water is impaired $(39,40)$, e.g., when stimulation of the antidiuretic hormone occurs. This may be triggered by medication, acute infection, stress, or by low solute intake in malnutrition/anorexia. The 2 patients who experienced hyponatremia had profound polydipsia characterized by a baseline 24-hour urinary output of more than $6 \mathrm{~L} / \mathrm{d}$. One patient received quetiapine, an antipsychotic drug known to predispose individuals to hyponatremia. The second patient had no other risk factors but did not respond to dulaglutide.

Guidelines for the management of primary polydipsia are lacking (5). To avoid complications, it seems sensible to minimize factors that impair the renal capacity to excrete water on the one hand, while on the other hand addressing the compulsive desire to drink liquids. A combination of pharmaceutical treatment (e.g., dulaglutide) and cognitive behavioral therapy might be a reasonable approach, but more research is needed in this area.

This trial has limitations. First, the 3-week treatment period was short, and we were unable to draw conclusions about the long-term effects of dulaglutide in primary polydipsia. Second, gastrointestinal symptoms and frequent side effects of GLP-1 RAs at the beginning of treatment may have interfered with the results. Reassuringly, during the evaluation visit and assessment of the primary endpoint, gastrointestinal symptoms were similar in both treatment arms, and the hypodipsic properties were also confirmed after adjusting for gastrointestinal adverse effects and accounting for treatment sequence. It is likely that the gastrointestinal symptoms at the start of treatment with dulaglutide compromised the blinding in this study. This had to be taken into consideration when interpreting the patient-reported secondary outcomes that were particularly prone to be affected by the knowledge of the assigned intervention. Third, we did not assess the primary outcome of "total fluid intake" at baseline, which lowers the confidence in the observed treatment effect. Fourth, dulaglutide is one of the larger GLP-1 RA molecules (>50-60 kDa) with possible impaired blood-brain barrier permeability (41). Therefore, our results are not directly transferrable to other GLP-1 RAs. However, smaller molecules with enhanced access to the central nervous system (e.g., liraglutide, semaglutide, or lixisenatide; ref. 42) may be even more potent in reducing fluid intake in primary polydipsia. Fifth, the fMRI substudy did not detect changes in functional brain activity despite clinically meaningful changes in the behavioral data. This is probably explained by the limited number and probably also by the non-thirsting state of the participants. Becker et al. (43), who used the same thirst-craving task in healthy controls, observed increased neural responses to beverage versus control pictures in a thirsting state (no drinking for 7 hours), but not in the no-thirst session.
The strength of our work is the prospective, double-blind, placebo-controlled trial design and the reasonably large cohort of patients with primary polydipsia. Given the high prevalence of psychiatric comorbidities, undertaking randomized controlled trials in patients with primary polydipsia is difficult and is also mirrored by the lack of research in this field (5).

In summary, our data show that a 3-week treatment with the GLP-1 RA dulaglutide reduced fluid intake, thirst perception, and voiding frequency in patients with primary polydipsia. This proofof-concept study is a first approach to provide a pharmaceutical treatment option for patients with primary polydipsia, for whom no effective treatment option currently exists.

\section{Methods}

\section{Study design and participants}

This was a single-center, randomized, double-blind, placebocontrolled, 3-week crossover trial conducted at the University Hospital Basel in Switzerland. A total of 50 participants (35 patients with primary polydipsia and 15 controls) were enrolled. Inclusion criteria for the patients in this study included age between 18 and 65 years and a diagnosis of primary polydipsia based on polydipsia of greater than $3000 \mathrm{~mL}$ per day and polyuria of greater than $50 \mathrm{~mL} / \mathrm{kg}$ body weight per day. Exclusion criteria were central or nephrogenic diabetes insipidus (excluded by water deprivation test or hypertonic saline test), secondary polyuria (e.g., diabetes mellitus, hypokalemia, hypercalcemia), inability to follow the study procedures, e.g., because of an unstable psychiatric condition, a history of pancreatitis, or treatment with a GLP-1 RA within the previous 3 months.

An exploratory fMRI case-controlled substudy was conducted in a subset of 15 patients and 15 matched controls. Exclusion criteria for the participation of patients in the fMRI substudy were any medical condition affecting the brain (e.g., stroke, epilepsy, multiple sclerosis), any ferromagnetic nonremovable device, and claustrophobia. Control participants were matched for age, sex, nicotine consumption, psychiatric comorbidities, and handedness. Matched controls underwent 1 fMRI session only and did not receive the study drug.

\section{Trial objective and outcomes}

The objective of this trial was to determine whether a 3-week treatment with the GLP-1 RA dulaglutide compared with placebo reduces fluid intake in patients with primary polydipsia. The primary outcome was total fluid intake (in $\mathrm{mL}$ ) during an 8-hour evaluation visit.

Further outcomes were thirst perception during the preceding weeks and during the evaluation visit, 24-hour urinary output, day- and nighttime voiding frequency, quality of life, serum and urine electrolytes and osmolality, and adverse effects, e.g., gastrointestinal symptoms.

The objective of the exploratory fMRI substudy was to assess whether dulaglutide as compared with placebo alters behavioral aspects (thirst rating) and the neuronal activity of patients while exposed to desirable beverage and control pictures. Further, we aimed to explore the effect of dulaglutide on the resting-state functional connectivity of homeostatic and reward-related brain regions.

\section{Trial procedures}

The trial procedures and timeline are schematically displayed in Supplemental Figure 8 . The allocation to treatment sequence was ran- 
domized 1:1 on the basis of a prefabricated randomization list of the Clinical Trial Unit Basel integrated into the electronic data capture system. Patients, investigators, and several nurses were blinded to the treatment arms, except for some unblinded study nurses, who injected the trial medication. Patients received a 3-week treatment with dulaglutide $(1.5 \mathrm{mg})$ or placebo ( $0.9 \%$ sodium chloride) subcutaneously once weekly and attended an 8-hour evaluation visit during the last treatment week. After a washout period of at least 3 weeks, patients received the complementary intervention. For the fMRI substudy, patients attended 2 additional visits and underwent an fMRI session during the last treatment week in each treatment phase.

Assessment of fluid intake and urinary output during the evaluation visit Patients arrived at 8:00 am at the trial site after a 12-hour overnight fast (no food or beverages). On arrival, the patients were asked to void their bladders, and a 24-hours urine collection was started. Clinical parameters and symptoms were assessed at 8:00 am, 12 $\mathrm{pm}$, and 4:00 pm. A 10 liter water dispenser was provided and refilled if necessary. Patients were invited to drink freely from the provided water, but they were blinded to the amount of water in the dispenser. At 8:30 am and at 12:00 pm, standardized, savory meals were provided (consisting of $116 \mathrm{~g}$ carbohydrates, $60 \mathrm{~g}$ fat, $25 \mathrm{~g}$ protein, $12 \mathrm{~g}$ fiber, and $5.7 \mathrm{~g}$ salt, for a total of $1284 \mathrm{kcal})$. To standardize for food intake, patients were asked to consume the entire meal, irrespective of their appetite. Besides the provided water and meals, no other beverages or food were allowed. At 4:00 pm, patients were asked to void their bladders and instructed to collect their urine until 8:00 am the following day.

\section{Assessment of thirst perception, drinking behavior, and electrolytes}

Thirst perception was assessed in 3 different ways: first, we assessed the average thirst perception of the preceding week at the weekly study visit using a 10-point NRS ( $0=$ no thirst, $10=$ extreme thirst). Second, during the evaluation visit, patients were asked at 8:00 am, 9:00 am, 12:00 pm, 1:00 pm, and 4:00 pm to indicate their current thirst perception on the 10-point NRS. Third, during the fMRI examination, thirst was rated while the participant was exposed to desirable beverage pictures as described below on a 7-point NRS.

Drinking behavior was assessed at the weekly study visits and included the reported average daily fluid intake and average daytime voiding frequency, as well as drinking at night and nocturia (yes/no) in the preceding week.

Electrolytes, glucose, and osmolality were assessed in plasma and urine at the screening visit as well as during the evaluation visits. Urinary electrolytes were also measured in the collected 24-hour urine.

\section{Assessment of quality of life}

We assessed quality of life in 2 ways at each weekly study visit and at the evaluation visit (relating to the timespan of the preceding week). First, patients were asked whether and how symptoms of polyuria and polydipsia (e.g., constant thirst, daytime voiding frequency, nocturia) had affected their quality of life on a 10-point NRS (i.e., 0 = quality of life not reduced, 10 = quality of life maximally reduced). Second, patients responded to the standardized short-form 12 (SF-12) questionnaire (44), a standardized questionnaire for the assessment of quality of life. Higher scores indicate a better state of health.

\section{Assessment of adverse effects}

At the weekly visit and during the evaluation visit, gastrointestinal symptoms, i.e. nausea and abdominal pain, were assessed on a 10-point NRS (e.g., 0 = no nausea, 10 = unbearable nausea). Other adverse effects were recorded in free text.

\section{fMRI}

Detailed information about the fMRI session methodology is provided in the Supplemental Methods. In brief, fMRI sessions consisted of a high-resolution structural image (T1) and 2 functional tasks (thirst rating task, resting state). The fMRI paradigm (43) showed pictures of beverages $(n=24)$ and chairs $(n=24)$ on a uniform gray-colored background. Pictures were pseudorandomized into 10 sets ( 5 sets of chairs and 5 sets of beverages) of 10 pictures, with each picture being shown for 2 seconds without interruption. After each set, patients had 4 seconds to rate their perceived thirst on a 7-point NRS. For standardization, patients abstained from drinking, eating, and smoking for at least 3 hours before the fMRI examination. The patient's serum sodium concentration was measured prior to the scan.

\section{Sample size estimation}

Sample size was estimated to show, with a power of $80 \%$, a $13 \%$ stronger decrease in the primary endpoint under dulaglutide as compared with placebo. Based on our own data, we assumed a mean (SD) fluid intake of $4630 \mathrm{~mL}(1710 \mathrm{~mL})$ before the start of treatment (45). We expected a decrease of $7 \%$ under placebo and $20 \%$ under dulaglutide. We used a random sampling procedure and examined sample sizes ranging from 20 to 120 patients by drawing 999 times each from a bivariate normal distribution with an assumed mean (SD) of 4.31 $(1.7 \mathrm{~L})$ after placebo and $3.7 \mathrm{~L}(1.7 \mathrm{~L})$ after dulaglutide, with a withinsubjects correlation of 0.8 . Using a Wilcoxon signed-rank test, the null hypothesis was rejected if the resulting $P$ value was lower than 0.05 . Accounting for a dropout rate of $15 \%$, a total of 35 patients would need to be recruited in order to have 29 evaluable patients.

\section{Statistics}

Statistical analyses were preplanned in a statistical report and analysis plan prior to database closure. The primary endpoint, and, unless indicated otherwise, all continuous secondary endpoints were analyzed for a treatment effect (dulaglutide versus placebo) using linear mixedeffect models (LMMs). First, the treatment sequence and interaction term between the trial arm and sequence were included to account for a carryover effect of the study drug. These analyses did not indicate a main effect of treatment sequence or an interaction effect of treatment. Therefore, the overall treatment effect was estimated. Detailed statistical methodology is described in the Supplemental Methods. Estimated treatment means and the mean difference are reported with a 95\% CI. The main analysis of the primary endpoint was performed as an intention-to-treat analysis including all patients (full analysis set). Further, a per-protocol analysis was performed on the basis of 31 patients who received all 3 injections in each trial phase. In addition, we performed sensitivity analyses, including adverse effects at the beginning and at any time point during the evaluation visit as additional covariates with an interaction term in the statistical model.

No missing values occurred for the primary endpoint. All secondary analyses were performed on complete cases of the full analysis set. 
Missing values were rare. We reported the number of available measurements for each endpoint.

Analyses were conducted using the statistics program $\mathrm{R}$, versions 3.6.0 and 4.0.2 (46). Linear mixed-effects models were fitted using the R package lme 4, and $P$ values were derived using the R package lmerTest. $P$ values and widths of CIs were not adjusted for multiplicity.

fMRI substudy analyses. No missing values occurred for the fMRI data. In patients, brain activation was compared between treatment (dulaglutide versus placebo) and stimuli (chair versus beverage) by means of an interaction analysis (treatment-stimuli interaction). The hypothesis was that dulaglutide diminishes the activation of thirstrelated areas during the presentation of beverage stimuli. Similarly, brain activation was compared between groups (patients on placebo versus controls) and stimuli (chair versus beverage) by means of an interaction analysis (group-stimuli interaction). We assumed that patients would have higher activation than controls in thirst-related areas such as the cingulate cortex, the insular cortex, or the amygdala while being exposed to beverage pictures.

In a subgroup analysis, only participants in a state of high thirst were analyzed ( $n=14$ patients on placebo and $n=10$ controls reporting a median thirst rating of 5 or more), assuming a higher activation of thirst-related areas during the presentation of beverage stimuli as compared with chairs (43).

We assessed the functional connectivity of 3 key regions of the reward network (left accumbens, right accumbens, midbrain) and the hypothalamus (Supplemental Figure 9) with the rest of the brain during the resting-state session (37). In patients, the main effect of treatment on functional connectivity values was tested with the hypothesis that functional connectivity between those core regions and other regions of the reward network would be altered on dulaglutide as compared with placebo (37). Similarly, we compared the functional connectivity of those regions between patients on placebo and control participants.

Details on the statistical analyses of the fMRI data are provided in the Supplemental Methods. Raw, unthresholded statistical maps are publicly available on NeuroVault (https://neurovault. org; https://identifiers.org/neurovault.collection:8995).

\section{Study approval}

The trial protocol and drug were approved by the ethics committee of North, West, and Central Switzerland (EKNZ, Basel, Switzerland) and the national agency for the authorization and supervision of therapeutic products (Swissmedic, Berne, Switzerland). Written informed consent was obtained from each participant after full explanation of the purpose and nature of all procedures used. The trial was registered with ClinicalTrials.gov (NCT02770885).

\section{Author contributions}

BW designed the study and wrote the protocol, collected, analyzed, and interpreted the data, conducted the literature search, and wrote the manuscript. COS was involved in the study design, collected, analyzed, and interpreted the data, conducted the literature search, and wrote the manuscript. DC analyzed and interpreted the fMRI data and contributed to the writing of the manuscript. DZ was involved in the study design and the setup of fMRI data acquisition. DRV planned, performed, and interpreted the statistical analyses and contributed to the writing of the manuscript. SAU and JR contributed to data collection. MCC designed the study, interpreted data, and supervised all steps of the conduct of the study. All authors edited and approved the final version of the manuscript. For the co-first authorship, BW is named first, as she initiated and designed the study.

\section{Acknowledgments}

We are grateful to our participants for taking part in the trial. We thank Christoph Becker and Harald Schupp for providing the fMRI paradigm. We thank support staff and the study and laboratory personnel at the University Hospital Basel, especially Nina Hutter, Cemile Bathelt, Joyce Santos de Jesus, Karin Wild, Silke Purschke, Vanessa Grassedonio, Klaus Ehrlich, Stefan Borgwardt, Francesco Santini, Tanja Haas, Philipp Madörin, Olivier Bieri, Nicole Bruni, Nica Jeanloz, Michelle Steinmetz, Katja Bologna, and Nicole Salvisberg for their most helpful support during the study. We thank the following organizations for their grant support: the Swiss National Foundation (SNF-162608, to MCC), the University Hospital Basel (to BW); the Young Talents in Clinical Research of the Swiss Academy of Medical Sciences and the G.\& J. Bangerter-Rhyner Foundation, and a top-up stipend from the $\mathrm{PhD}$ Program Health Sciences, University Hospital Basel, Switzerland (to COS).

Address correspondence to: Bettina Winzeler, University Hospital Basel, Department of Endocrinology, Diabetology und Metabolism, Petersgraben 4, 4031 Basel, Switzerland. Phone: 41.61.556.50.75; Email: bettina.winzeler@usb.ch.
1. Goldman MB, et al. Mechanisms of altered water metabolism in psychotic patients with polydipsia and hyponatremia. N Engl JMed. 1988;318(7):397-403.

2. Barlow ED, De Wardener HE. Compulsive water drinking. QJMed. 1959;28(110):235-258.

3. de Leon J, et al. Polydipsia and water intoxication in psychiatric patients: a review of the epidemiological literature. Biol Psychiatry. 1994;35(6):408-419.

4. Sailer C, et al. Primary polydipsia in the medical and psychiatric patient: characteristics, complications and therapy. Swiss Med Wkly. 2017;147:w14514.

5. Brookes G, Ahmed AG. Pharmacological treatments for psychosis-related polydipsia. Cochrane
Database Syst Rev. 2006;(4):CD003544.

6. Verghese C, et al. Problems and progress in the diagnosis and treatment of polydipsia and hyponatremia. Schizophr Bull. 1996;22(3):455-464.

7. Bremner AJ, Regan A. Intoxicated by water. Polydipsia and water intoxication in a mental handicap hospital. Br J Psychiatry. 1991;158:244-250.

8. Alexander RC, et al. A double blind, placebocontrolled trial of demeclocycline treatment of polydipsia-hyponatremia in chronically psychotic patients. Biol Psychiatry. 1991;30(4):417-420.

9. de Leon J, et al. Treatment of polydipsia and hyponatremia in psychiatric patients. Can clozapine be a new option? Neuropsychopharmacology. 1995;12(2):133-138.
10. Bak M, et al. Almost all antipsychotics result in weight gain: a meta-analysis. PLoS One. 2014;9(4):e94112.

11. Gutniak M, et al. Antidiabetogenic effect of glucagon-like peptide-1 (7-36)amide in normal subjects and patients with diabetes mellitus. N Engl J Med. 1992;326(20):1316-1322.

12. Nauck MA, et al. Normalization of fasting hyperglycaemia by exogenous glucagon-like peptide 1 (7-36 amide) in type 2 (non-insulin-dependent) diabetic patients. Diabetologia. 1993;36(8):741-744.

13. Smith GP, Gibbs J. Brain-gut peptides and the control of food intake. Adv Biochem Psychopharmacol. 1981;28:389-395.

14. Turton MD, et al. A role for glucagon-like pep- 
tide-1 in the central regulation of feeding. Nature. 1996;379(6560):69-72.

15. Andersen A, et al. Glucagon-like peptide 1 in health and disease. Nat Rev Endocrinol. 2018;14(7):390-403.

16. Gutzwiller JP, et al. Glucagon-like peptide-1 is involved in sodium and water homeostasis in humans. Digestion. 2006;73(2-3):142-150.

17. McKay NJ, et al. Endogenous glucagon-like peptide-1 reduces drinking behavior and is differentially engaged by water and food intakes in rats. J Neurosci. 2014;34(49):16417-16423.

18. McKay NJ, Daniels D. Glucagon-like peptide-1 receptor agonist administration suppresses both water and saline intake in rats. J Neuroendocrinol. 2013;25(10):929-938.

19. Tang-Christensen $M$, et al. Central administration of GLP-1-(7-36) amide inhibits food and water intake in rats. Am J Physiol. 1996;271(4 pt 2):R848-R856.

20. Denton DA, et al. Hypothalamic integration of body fluid regulation. Proc Natl Acad Sci U S A. 1996;93(14):7397-7404.

21. McKinley MJ, et al. Physiological and pathophysiological influences on thirst. Physiol Behav. 2004;81(5):795-803.

22. Augustine V, et al. Hierarchical neural architecture underlying thirst regulation. Nature. 2018;555(7695):204-209.

23. McKay NJ, et al. Glucagon-like peptide-1 receptor agonists suppress water intake independent of effects on food intake. Am J Physiol Regul Integr Comp Physiol. 2011;301(6):R1755-R1764.

24. Winzeler B, et al. Effects of glucagon-like peptide-1 receptor agonists on fluid intake in healthy volunteers. Endocrine. 2020;70(2):292-298.

25. Engel JA, Jerlhag E. Role of appetite-regulating peptides in the pathophysiology of addiction: implications for pharmacotherapy. CNS Drugs. 2014;28(10):875-886.
26. Hayes MR, Schmidt HD. GLP-1 influences food and drug reward. Curr Opin Behav Sci. 2016;9:66-70.

27. McKinley MJ, et al. From sensory circumventricular organs to cerebral cortex: Neural pathways controlling thirst and hunger. J Neuroendocrinol. 2019;(3):e12689.

28. Saker P, et al. Influence of anterior midcingulate cortex on drinking behavior during thirst and following satiation. Proc Natl Acad Sci US A. 2018;115(4):786-791.

29. Pool AH, et al. The cellular basis of distinct thirst modalities. Nature. 2020;588(7836):112-117.

30. Augustine V, et al. Neural control and modulation of thirst, sodium appetite, and hunger. Cell. 2020;180(1):25-32.

31. Barson JR, et al. Similarities in hypothalamic and mesocorticolimbic circuits regulating the overconsumption of food and alcohol. Physiol Behav. 2011;104(1):128-137.

32. Larsen PJ, et al. Distribution of glucagon-like peptide- 1 and other preproglucagon-derived peptides in the rat hypothalamus and brainstem. Neuroscience. 1997;77(1):257-270.

33. Merchenthaler I, et al. Distribution of pre-pro-glucagon and glucagon-like peptide-1 receptor messenger RNAs in the rat central nervous system. J Comp Neurol. 1999;403(2):261-280.

34. Alvarez E, et al. Expression of the glucagon-like peptide-1 receptor gene in rat brain. J Neurochem. 1996;66(3):920-927.

35. Zanchi D, et al. The impact of gut hormones on the neural circuit of appetite and satiety: a systematic review. Neurosci Biobehav Rev. 2017;80:457-475.

36. De Silva A, et al. The gut hormones PYY 3-36 and GLP-1 7-36 amide reduce food intake and modulate brain activity in appetite centers in humans. Cell Metab. 2011;14(5):700-706.

37. Meyer-Gerspach AC, et al. Endogenous GLP-1 alters postprandial functional connectivity between homeostatic and reward-related brain regions involved in regulation of appetite in healthy lean males: A pilotstudy. Diabetes Obes Metab. 2018;20(10):2330-2338.

38. Sailer CO, et al. Characteristics and outcomes of patients with profound hyponatraemia due to primary polydipsia. Clin Endocrinol (Oxf). 2017;87(5):492-499.

39. Musch W, et al. Solute loss plays a major role in polydipsia-related hyponatraemia of both water drinkers and beer drinkers. QJM. 2003;96(6):421-426.

40. Berl T. Impact of solute intake on urine flow and water excretion. JAm Soc Nephrol. 2008;19(6):1076-1078.

41. Glaesner W, et al. Engineering and characterization of the long-acting glucagon-like peptide-1 analogue LY2189265, an Fc fusion protein. Diabetes Metab Res Rev. 2010;26(4):287-296.

42. Hunter K, Holscher C. Drugs developed to treat diabetes, liraglutide and lixisenatide, cross the blood brain barrier and enhance neurogenesis. BMC Neurosci. 2012;13:33.

43. Becker CA, et al. Thirst and the state-dependent representation of incentive stimulus value in human motive circuitry. Soc Cogn Affect Neurosci. 2015;10(12):1722-1729.

44. Ware J, et al. A 12-Item Short-Form Health Survey: construction of scales and preliminary tests of reliability and validity. Med Care. 1996;34(3):220-233.

45. Timper K, et al. Diagnostic accuracy of copeptin in the differential diagnosis of the polyuria-polydipsia syndrome: a prospective multicenter study. J Clin Endocrinol Metab. 2015;100(6):2268-2274.

46. R: A Language and Environment for Statistical Computing. Version 4.1.1. R Foundation; 2021. Accessed September 3, 2021. https:// www.r-project.org/. 